\title{
Collective Phenomena in Complex Social Networks
}

Federico Vazquez, Juan Carlos González-Avella, Víctor M. Eguíluz and Maxi San Miguel.

\begin{abstract}
The problem of social consensus is approached from the perspective of nonlinear dynamics of interacting agents in a complex network. Some basic concepts, such as dynamical metastability, are discussed in the framework of the prototype voter model. In the context of Axelrod's model for the dissemination of culture we describe a co-evolutionary dynamics formulation with recent results on group formation and nonequilibrium network fragmentation and recombination transitions.
\end{abstract}

\section{Introduction}

Nonlinear dynamics includes a set of concepts, tools and generic phenomena that are being successfully applied to understand complex behavior of natural systems and man made devices. From this understanding new designs for improved technology are constantly being proposed. The study of social dynamics from this perspective of nonlinear phenomena is a relatively new scientific challenge attracting an increasing number of researchers coming from different disciplines. But also here, the under-

Federico Vazquez

IFISC, Instituto de Física Interdisicplinar y Sistemas Complejos (CSIC-UIB), E-07122 Palma de Mallorca, Spain, e-mail: federico@ifisc.uib.es

Juan Carlos González-Avella

IFISC, Instituto de Física Interdisicplinar y Sistemas Complejos (CSIC-UIB), E-07122 Palma de Mallorca, Spain, e-mail: juancarlos@ifisc.uib.es

Víctor M. Eguíluz

IFISC, Instituto de Física Interdisicplinar y Sistemas Complejos (CSIC-UIB), E-07122 Palma de Mallorca, Spain, e-mail: victor@ifisc.uib.es

Maxi San Miguel

IFISC, Instituto de Física Interdisicplinar y Sistemas Complejos (CSIC-UIB), E-07122 Palma de Mallorca, Spain, e-mail: maxi@ifisc.uib.es 
standing of basic mechanisms operating in the nonlinear social interactions should pave the way to innovative proposals to handle conflicts arising in the man made system which is a society.

Collective phenomena emerge from the nonlinear interactions of individual agents. The existence of thresholds or transitions between two collective states reflect an underlying nonlinear dynamics. A general class of social collective phenomena fall within what is termed consensus problems: The question is to establish when the dynamics of a set of interacting agents that can choose among several options (political vote, opinion, language, cultural features, etc.) leads to a consensus in one of these options, or when a state with several coexisting social options prevails. The former is an ordered state, while the latter is called a polarized state. An important issue is to identify nonlinear mechanisms producing a polarized state in spite of general convergent dynamics. The interaction among agents has two different ingredients. The first is the mechanism by which agents interact: the interaction rule. It can be an imitation mechanism, a social pressure mechanism (following a majority of neighbors), following a leader, etc. The second ingredient is the social network, that is, who interacts with whom. The process of formation of complex social networks is itself a nonlinear process. It turns out that the fact that a consensus is reached or not depends on nontrivial ways on both ingredients.

In this paper we address the consensus problem from the point of view of nonlinear interactions in complex networks. We first consider the prototype voter model $[1,2]$ of imitation dynamics for agents with two possible options. We describe a phenomena of dynamic metastability that occurs in complex networks of high dimensionality. There are long lived polarized states in which spatial domains of the two options coexist. Secondly, we consider a model proposed by Axelrod [3] for the dissemination of culture. This model can be considered as a multivariable elaboration of the voter model dynamics. This model, in a fixed network of interactions, exhibits a nonequilibrium transition between the ordered and polarized states for a critical value of the parameter measuring initial cultural diversity. We analyze here how this phenomena is modified when there is coupled dynamics (co-evolution) of the state of the agents and the links defining the social network. This results in a complex dynamics of group formation with nonequilibrium network fragmentation and recombination transitions.

\section{Voter Model}

The voter model is a stochastic model originated in the mathematical literature [1,2] recently considered in a variety of situations $[6,7,8,9,10]$. It is defined by a set of agents with two possible opinions or spins $s_{i}= \pm 1$ located at the nodes of a network. The elementary dynamical step in a node-update dynamics consists in randomly choosing one node and assigning to it the opinion, or spin value, of one of its neighbors, also chosen at random. A neighbor is defined as an agent connected to 
the one being considered by a link of the network. This interaction rule implements a simple mechanism of social imitation.

The voter model dynamics has two absorbing states, corresponding to situations in which all the spins have converged to the $s_{i}=1$ or to the $s_{i}=-1$ consensus states. The ordering dynamics is stochastic and driven by interfacial noise. A standard order parameter to describe the ordering process $[6,7]$ is the average of the interface density $\rho$, defined as the density of links connecting nodes with different spin value. In a disordered configuration with randomly distributed spins $\rho \simeq 1 / 2$, while for a completely ordered system $\rho=0$. In regular lattices of dimensionality $d \leq 2$ the system orders. This means that, in an infinite large system, there is a coarsening process with unbounded growth of spatial domains of one of the two opinions. The asymptotic regime of approach to the ordered state is characterized in $d=1$ by a power law $\langle\rho\rangle \sim t^{-\frac{1}{2}}$, while for the critical dimension $d=2$ a logarithmic decay is found $\langle\rho\rangle \sim(\ln t)^{-1}[4,6]$. Here the average $\langle\cdot\rangle$ is an ensemble average.

Intuition might indicate that consensus should be reached more efficiently modifying a regular network by introducing random links that connect far away nodes of the network (that is, creating a small-world network [11]). It turns out that this intuition is wrong. For networks with random connections (and also for regular lattices with $d>2$ ), the voter dynamics does not order the system in the thermodynamic limit of large systems $[5,7,8,10]$. Starting from a random initial condition and after an initial transient, the system falls in a partially ordered dynamical metastable state. After the initial transient $\rho$ fluctuates randomly around an average plateau value $\xi$ which gives the characteristic linear dimension of an ordered domain of one of the two options. In a finite system the metastable state has a finite lifetime: a finite size fluctuation takes the system from the metastable state to one of the two ordered absorbing states. In this process the fluctuation orders the system and $\rho$ changes from its metastable plateau value to $\rho=0$ (see Fig. 1). Note that these dynamical metastable states are different from frozen states that occur even in finite systems in other dynamical models.

Fig. 1 Interface density evolution for several realizations of voter model dynamics in a random network.

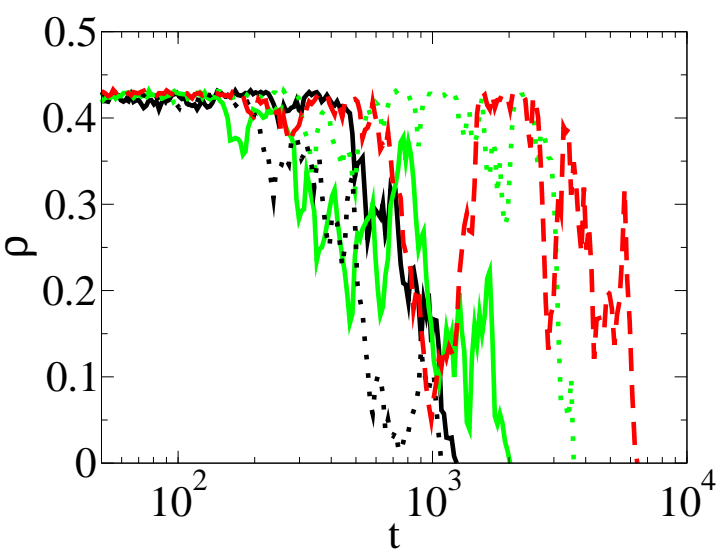


Fig. 2 Time evolution of the mean interface density for three random networks: Barabasi-Albert (circles),

Exponential (squares) and Erdős-Rényi (diamonds). The system size is $N=10^{4}$ and the average connectivity is $\langle k\rangle=8$. The continuous line gives the mean-field result. Numerical simulations depart from this result due to finite size effects.

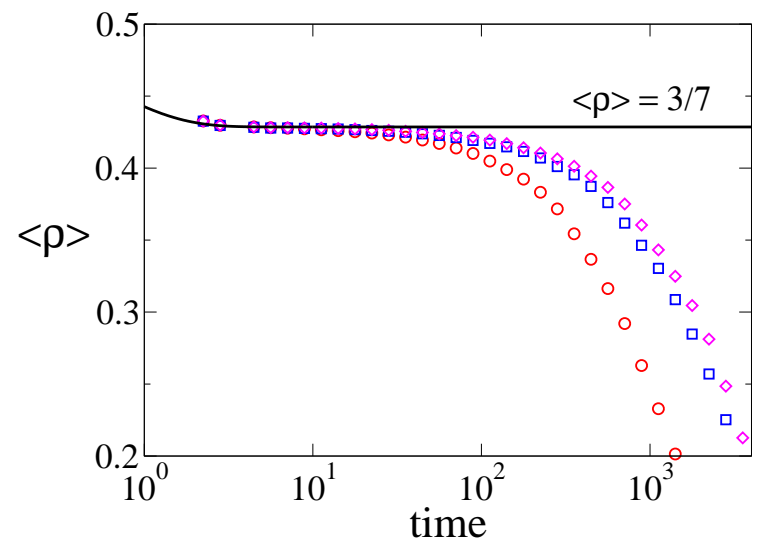

Such metastable states can be characterized within a mean field approximation focusing on the link dynamics. One finds that for a random network and in the limit of infinite system size

$$
\xi=\frac{\langle k\rangle-2}{2(\langle k\rangle-1)}
$$

where $\langle k\rangle$ is the average degree of the network, that is the average number of neighbors of a node. This result [21] establishes a one parameter theory in which the degree of order reached by the system is determined only by $\langle k\rangle$ in any random network. Fig. 2 checks this result against numerical simulations for different random networks. The exponential random network is constructed as in the Barabasi network [12] but with random instead of preferential attachment of new nodes, therefore it has an exponential degree distribution. In the Erdős-Rényi network every pair of nodes is connected by a link with probability $p$, giving a Poisson degree distribution with average connectivity $\langle k\rangle=N p$.

\section{Axelrod Model}

One of the crucial and yet unanswered questions in the social sciences is: if people become more similar in their beliefs, attitudes and behavior as they interact, why doesn't this interaction lead to homogeneity? [3].

To answer this question, social scientists have been proposing various mechanisms. A few years ago Robert Axelrod introduced a model [3] that explores the competition between consensus and polarization, in an attempt to describe how the culture disseminates within a society. He proposed a mechanism in which people become more alike when they interact (social influence) as, for instance in the voter model $[1,2]$, but now with the possibility that the tendency to converge stops before it reaches completion. It also incorporates the idea that partners with more similarities interact more frequently than those with less similarities (homophily). 
The "culture" of an individual is defined as a set of $F$ different attributes, each of which can assume one of $q$ possible traits, and they describe the individual's features. For instance, for $F=3$ features and $q=4$ traits per feature, we could have:

1. Language: English, Spanish, French, Hebrew;

2. Religion: Protestant, Catholic, Muslim, Jewish;

3. Food preference: pasta, meat, vegetables, tofu.

The basic mechanism is that neighbors who share at least one feature are more likely to interact, after which they agree on another feature previously not shared, thus becoming even more similar; while neighbors with no features in common do not interact.

This model has attracted the attention of many statistical physicists due to its rich dynamics and non-trivial behavior. It belongs to the class of stochastic models that, started from a given initial state, may or may not reach the consensus state. This gives rise to its most intriguing feature: an order-disorder non-equilibrium phase transition as we shall explain.

\subsection{Model definition and results on a square lattice}

The mathematical model consists of a population of individuals located at the nodes of a 2D square lattice that can interact only with its first four neighbors. The culture of an individual $i$ is represented by an $F$-component array $C_{i}^{f}(f=1,2 \ldots, F)$ where each component can take one of $q$ different values $\left(C_{i}^{f}=0,1 \ldots, q-1\right)$. There are, therefore, $q^{F}$ different possible states or type of individuals.

At each time step one individual $i$ is chosen at random, and then it randomly picks one of its neighbors $(j)$. Then one feature $f$ is chosen randomly: if $C_{i}^{f} \neq C_{j}^{f}$ nothing happens but if $C_{i}^{f}=C_{j}^{f}$ another feature $f^{\prime}$ among the non-shared ones is chosen randomly and individual $i$ adopts individual's $j$ feature $\left(C_{i}^{f^{\prime}}=C_{j}^{f^{\prime}}\right)$. This last step is equivalent to making $i$ and $j$ interact with a probability equal to the fraction of features that they share $m / F$, where $m=\sum_{f=1}^{F} \delta_{C_{i}^{f}, C_{j}^{f}}$ is their overlap and $\delta$ is the Kronecker delta function.

The model posses two competing features that lead to a very rich dynamical behavior. On the one hand, the interactions between nearest neighbors lead to more similarity producing the formation of same-culture domains. On the other hand, the incompatibility constrained between totally different neighbors prevents global consensus.

For a finite population, the system eventually freezes in a configuration that depends on $q$. For small $q$, the system coarsens as the neighbors tend to share the same culture and it freezes in a state where the average size of the largest monocultural region $\left\langle S_{\max }\right\rangle$ is similar to the system size $N$ (monocultural phase) (see fig. 3). As $q$ is increased beyond a critical value $q_{c},\left\langle S_{\max }\right\rangle / N$ suddenly drops to zero meaning 
Fig. 3 Average size of the largest cultural domain $\left\langle S_{\max }\right\rangle$ vs $q$ for $F=10$ features and system sizes $N=900$ (circles), $N=1600$ (squares) and $N=2500$ (diamonds). The transition at the critical point $q_{c} \simeq 55$ becomes sharper as the system size increases.

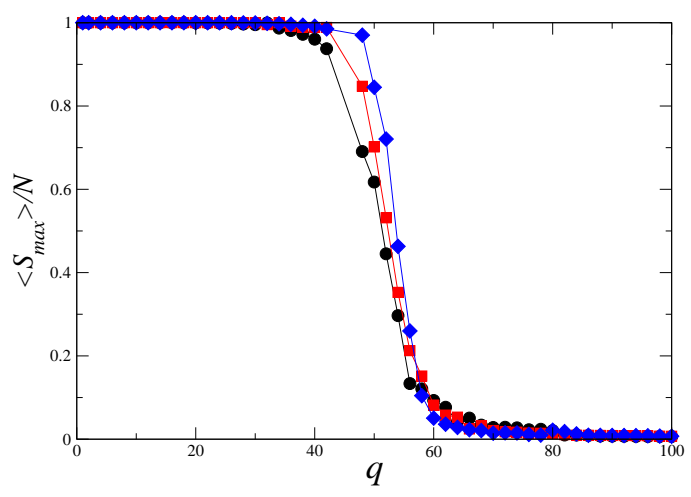

that in the final state all the regions have a size much smaller than $N$ (multicultural phase).

\subsection{The model in a co-evolving network}

In section 3.1 we presented the original version of the model, where individuals are assumed to interact always with the same neighbors. However, real-life experience shows that people have a tendency to select their interacting partners according to their evolving social preferences. In this section we present a version of the model in which the interacting neighborhood of individuals changes with time. Individuals drop their ties with incompatible partners and form new ties with other like-minded individuals.

Individuals are located at the nodes of a degree-regular graph where every node has exactly $\langle k\rangle$ neighbors, and they initially take values for the features at random from 0 to $q-1$. In a Monte Carlo step, an individual $i$ and one of its neighbors $j$ are randomly chosen. If their overlap $m$ is larger than zero they interact with probability

Fig. 4 Node degree distribution $P(k)$ of the co-evolving network in the final frozen state for a system with $F=10$ features, population size $N=10^{4}$ and various values of $q$. The system starts from random network with average degree $\langle k\rangle=4 . P(k)$ is very similar to a Poissonian (sketched in empty squares for comparison) for all values of $q$.

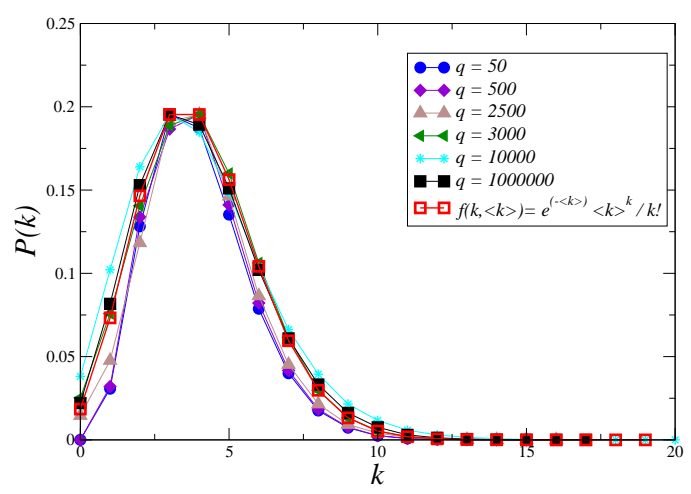


$m / F$ (as in the original model) and, in case of an interaction, the overlap is increased by one. However, if the overlap is zero, then $i$ removes its link to $j$ and attaches it to a randomly chosen node $l$ with the condition that $l$ was not already a neighbor of $i$.

Due to the random rewiring dynamics the original network quickly evolves into a random graph with a Poisson degree distribution with average connectivity $\langle k\rangle$. This distribution is conserved until the system freezes (see fig. 4).

Even though the node degree distribution seems to be independent on $q$, the structure of the network in the final frozen state dramatically depends on $q$. During the evolution nodes are grouped in different communities with individuals sharing some features. Thus the network gets disconnected in different network components of like-minded individuals. Inside a component there are also groups of nodes with the same state, that we call domains.

In order to characterize the final structure of the network we calculated the average size of the largest component $S$ as a function of $q$ for simulations on a population of $N=2500$ individuals and $F=3$ features (fig. 5).

We observe that there are two transitions in the absorbing state: a fragmentation transition from phase $I$ to phase $I I$ and a recombination transition from phase $I I$ to phase $I I I$. In phases $I$ and $I I$, the system is frozen and composed by a set of disconnected components whose size depends on the degree of initial diversity $q$. For values of $q$ smaller than a critical value $q_{c} \simeq 85$ (phase $I$ ), $S$ is similar to the system size $N$ (figs. 6-a and 6-b), while for $q$ above $q_{c}$ (phase $I I$ ), $S$ is much smaller than $N$ (fig. 6-c). In analogy to the characterization of non-equilibrium phase transitions, we define $q_{c}$ as the the value of $q$ where the fluctuations in $S$ reach a maximum value. The critical value $q_{c} \simeq 85$ in this co-evolving network is larger that the corresponding value $q_{c} \simeq 55$ obtained in a fixed $2 \mathrm{D}$ square lattice (Fig. 3 ). When $q$ is above a transition point $q^{*}$ (phase $I I I$ ), the system reaches a dynamically active configuration with many small domains. These domains have zero overlap between them and they are interconnected by links making up a large network component compared to the system size, as we observe in fig. 6-d. Above the value $q^{*} \simeq \frac{N F}{\langle k\rangle}$ the

Fig. 5 Average relative size of the largest network component (circles) and largest domain $\left\langle S_{\max }\right\rangle$ (solid line) in the stationary configuration vs $q$, for $F=3$ features, $N=2500$, averaged over 400 realizations. The vertical lines at $q_{c}=85$ and $q^{*}=1875$ indicate the transition points between the different phases.

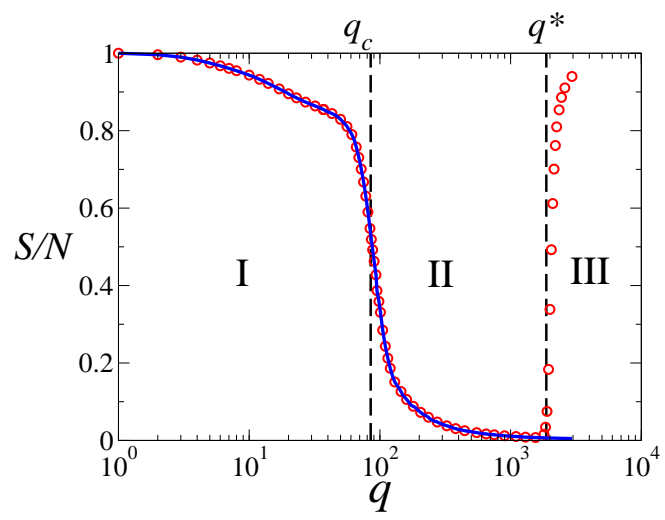




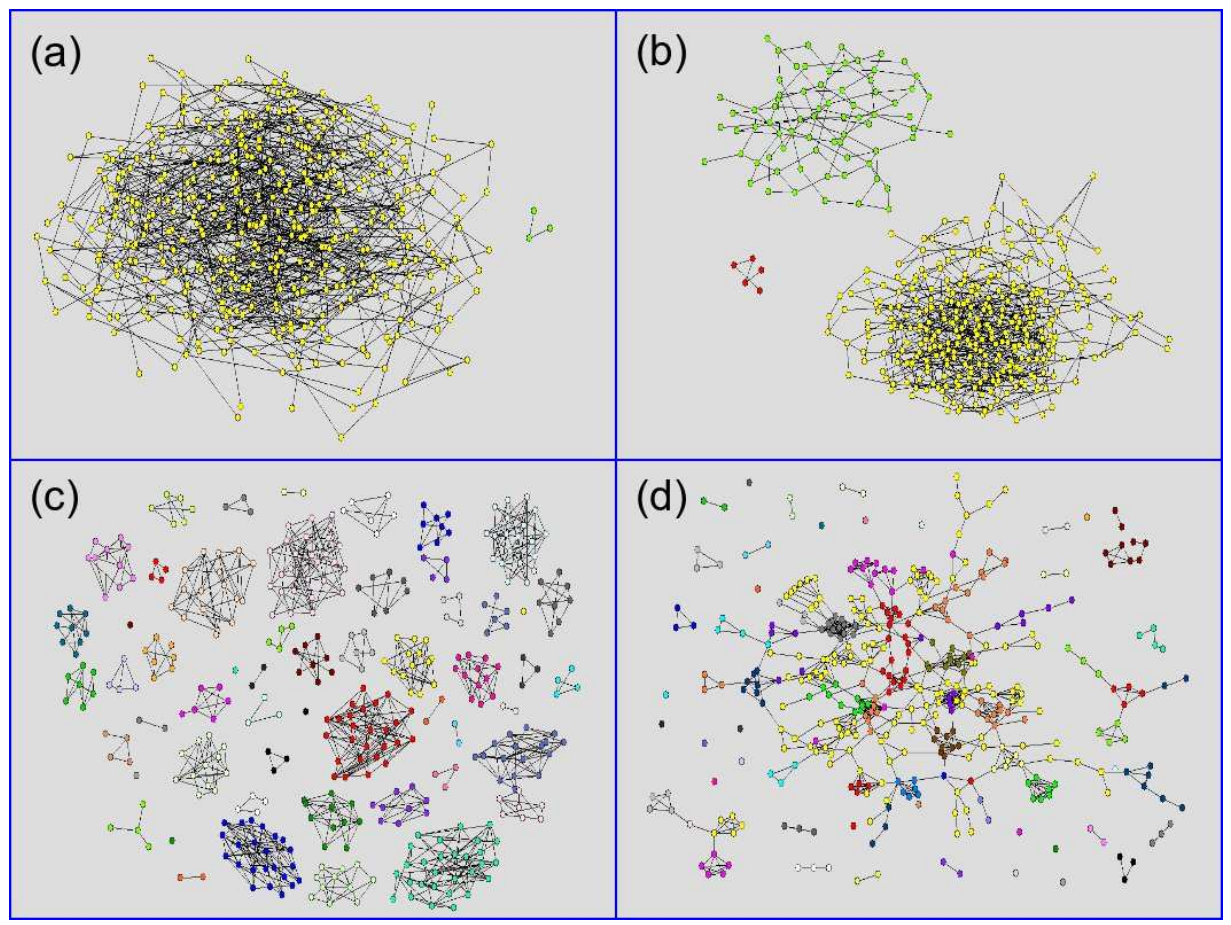

Fig. 6 Network structure in the final frozen configuration in phase $I: q=3$ (a), $q=20$ (b) and in phase II: $q=100$ (c) for $N=400$. (d) Snapshot of the network in the stationary active configuration (phase $I I I$ ) for $q=500$.

initial number of pairs of nodes with at least one feature in common is smaller than the number of links in the system.

The nature of both transitions can be understood by studying the dynamical approach of the system to the final state in the three phases (see [20] for details).

\subsubsection{Changing the rewiring rate}

In the previous section we assumed that when a pair of nodes with zero overlap is chosen, the link between them is always rewired. We now consider the case in which the rewiring happens with probability $p$. Varying $p$ is equivalent to change the relative time scales at which the copy and the rewiring dynamics occur. In the limit of $p$ going to zero we expect the system to behave as in the original Axelrod model, where the network is fixed. On the other limit, when $p$ is one we recover the co-evolving model studied before. Thus, we should see that the transition point $q_{c}$ shifts to higher values of $q$ as $p$ is increased from zero. Fig. 7 shows that the critical point for values of $p$ above 0.1 is very close to the critical value $p_{c} \simeq 85$ for the $p=1$ case, when $S$ is measured at a fixed time $\tau=10^{8}$. To investigate this dependence, 
Fig. 7 Relative size of the largest domain vs $q$ for values of the rewiring probability $p=0,10^{-6}, 5 \times 10^{-5}, 0.1$ and 1 from left to right, $N=1026$ and $F=3$ features. $S$ was measured for every value of $p$ at the same observation time $\tau=10^{8}$.

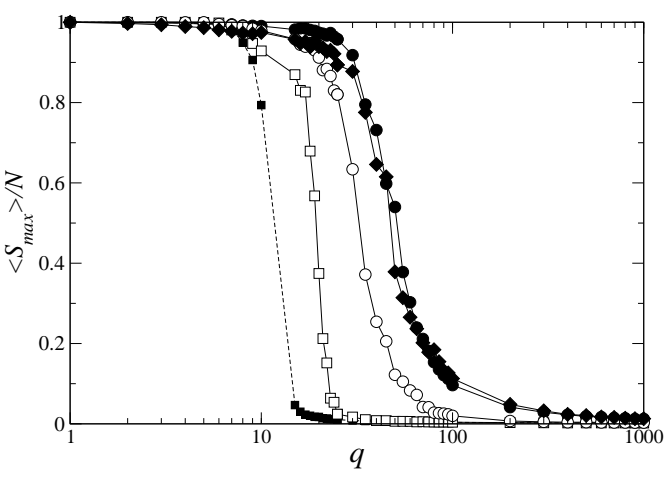

we calculated $S$ for fixed $q=20$ in the connected phase $I$, and for three different observation times. As we observe in fig. 8, for a fixed time, $S / N$ is close to zero for very small values of $p$ and it increases up to $S / N \simeq 0.9$ for larger values of $p$ when the observation times are $\tau=10^{8}$ and $\tau=10^{11}$. However this transition in $S$ seems to disappear when $S$ is measured at longer times $\left(\tau=10^{13}\right)$. We expect that if we wait long enough the size of the largest component would reach the value $S / N \simeq 0.9$ for any value of $p$ above zero. The last result means that there is a discontinuity in the critical value $q_{c}$ at $p=0$. For any $p>0$, the behavior of $S$ as a function of $q$ is essentially the same in the long time limit.

\subsection{Summary}

The voter model for opinion formation exhibits a non-trivial dynamical behavior that depends on the spatial dimension of the interacting network of voters. In a finite network the system always reaches the consensus state due to fine size fluctuations. However, in infinite large networks, the consensus depends on the dimension. For

Fig. 8 Relative size of the largest domain vs the rewiring probability $p$ for $N=1024$, $F=3, q=20$ and observation times $\tau=10^{8}$ (squares), $\tau=10^{11}$ (diamonds) and $\tau=10^{13}$ (circles). For a long enough observation time, $\left\langle S_{\max }\right\rangle / N$ approaches to the value $\left\langle S_{\max }\right\rangle / N \simeq 0.9$ independent on the value of $p$.

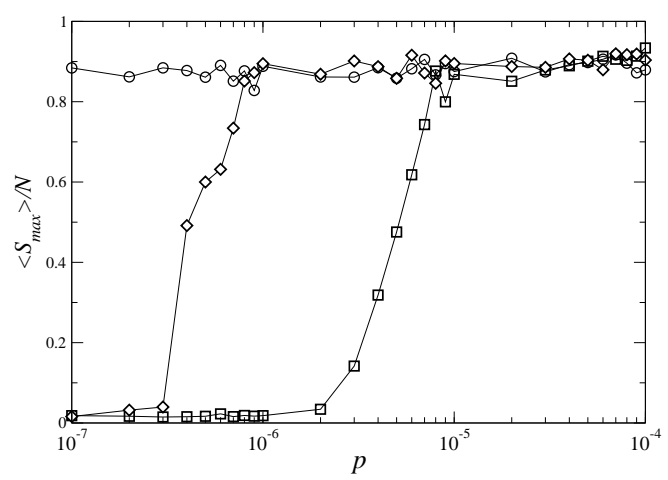


$d \leq 2$ consensus is always reached in a characteristic time that depends on $d$. For $d>$ 2 , including random networks, the system reaches a stationary state where the two opinions coexist. This state is characterized by the average value of the stationary density of interfaces that monotonically increases with the average connectivity of the network.

The Axelrod model for the dissemination of culture is an incredibly rich nonequilibrium model that posses a quite interesting nonlinear dynamics and it is simple enough to be treated with traditional statistical physics tools. A phase transition from an ordered to a disordered phase is found when the degree of initial diversity in the population is increased above a critical value. When a co-evolutionary dynamics is added to the model, the order-disorder transition still happens but at a larger value $q_{c}$. The network remains connected below $q_{c}$, but it disintegrates in multiple small components above $q_{c}$ (fragmentation). There is also a second transition at a much larger value $q^{*}$ where a giant network component grows again as $q$ is increased above $q^{*}$ (recombination). We have also found that changing the rewiring rate does not affect the final result on co-evolution, it just adds an overall time scale to the system. The robustness of the co-evolving model was checked in [19] under the influence of an external noise. While in a fixed network the addition of a small amount of noise has the effect of ordering the system, in a co-evolving network the diversity is sustained even under the influence of a small external noise; i.e, the co-evolving Axelrod model is robust.

Acknowledgements We acknowledge financial support from MEC (Spain) through projects CONOCE2 (FIS2004-00953) and FISICOS (FIS2007-60327).

\section{References}

1. Holley R. Liggett T., Ann. Probab. 4, 1975, 195.

2. T.M. Liggett, Interacting Particle Systems (Springer, New York 1985).

3. R. Axelrod, The dissemination of culture: A model with local convergence and global polarization, J. Conflict Res. 41, 203-226 (1997).

4. L. Frachebourg and P.L. Krapivsky, Exact results for kinetics of catalytic reactions, Phys. Rev. E 53, R3009-3012 (1996).

5. P.L. Krapivsky, Kinetics of monomer-monomer surface catalytic reactions, Phys. Rev. A 45, 1067-1072 (1992).

6. I. Dornic, H. Chaté, J. Chavé, and H. Hinrichsen, Critical Coarsening without Surface Tension: The Universality Class of the Voter Model, Phys. Rev. Lett. 87, 045701-045074 (2001).

7. C. Castellano, D. Vilone, and A. Vespignani,Incomplete ordering of the voter model on smallworld networks, Europhysics Letters 63, 153-158 (2003).

8. K. Suchecki, V. M. Eguíluz, and M. San Miguel, Conservation laws for the voter model in complex networks, Europhysics Letters 69, 228-234 (2005).

9. V. Sood and S. Redner, Voter Model on Heterogeneous Graphs, Phys. Rev. Lett. 94, 178701178704 (2005).

10. K. Suchecki, V. M. Eguíluz, and M. San Miguel, Voter model dynamics in complex networks: Role of dimensionality, disorder and degree distribution, Phys. Rev. E (2005)

11. D.J. Watts and S.H. Strogatz, Collective dynamics of 'small-world' networks, Nature 393, 440-443 (1998). 
12. A. L. Barabási and R. Albert, Emergence of Scaling in Random Networks, Science 286, 509512 (1999).

13. C. Castellano, M. Marsili, A Vespignani, Nonequilibrium Phase Transition in a Model for Social Influence, Phys. Rev. Lett. 85, 3536-3539 (2000).

14. K. Klemm, V.M. Eguíluz, R. Toral, and M. San Miguel, Role of dimensionality in Axelrod's model for the dissemination of culture, Physica A 327, 1-5 (2003).

15. D. Vilone, A. Vespignani, and C. Castellano, Ordering phase transition in the one-dimensional Axelrod model, Eur. Phys. J. B 30, 399-406 (2002).

16. K. Klemm, V.M. Eguíluz, R. Toral, and M. San Miguel,Globalization, polarization and cultural drift, J. Econ. Dyn. Control 29, 321-334 (2005).

17. K. Klemm, V.M. Eguíluz, R. Toral, and M. San Miguel, Nonequilibrium transitions in complex networks: a model of social interaction, Phys. Rev. E 67, 026120(1-6) (2003).

18. K. Klemm, V.M. Eguíluz, R. Toral, and M. San Miguel,Global culture: A noise indcued transition in finite systems, Phys. Rev. E 67, 045101(1-4)(R) (2003).

19. D. Centola, J. C. Gonzalez-Avella, V. M. Eguiluz , and M. San Miguel, J. Conflict Resolution (2007)

20. F. Vazquez, J. C. Gonzalez-Avella, V. M. Eguiluz and M. San Miguel, Phys. Rev. E (2007), arXiv:0708.0776.

21. F. Vazquez, V. M. Eguiluz, and M. San Miguel, unpublished. 